\title{
Research on Design Practice of PLC in Automatic Control System of Electrical Equipment
}

\author{
Zhaosen Zhang, Yan Gao, Chen Ye \\ Yangzhou Polytechnic College, Yangzhou, Jiangsu, 225009, China
}

\begin{abstract}
PLC control system has a very wide range of applications in today's electrical automation equipment, because it has the characteristics of good stability, strong environmental adaptability, low cost and diversified program editing according to actual needs, making it automatic The efficiency of control has been greatly improved. Based on the current development of electrical equipment automation in China, this paper analyzes the effects and characteristics of PLC used in automation control systems from many aspects. It studies the selection and application of PLC in the operation of electrical equipment, and proposes to improve production efficiency. Some feasible measures.
\end{abstract}

Keywords: Electrical equipment; automatic control; PLC system; design practice

The whole process of PLC is an editable logic controller. It is an important device for effective resource allocation of current electrical equipment and automatic operation of electrical equipment. It is the most important part of the automation control system. Stable and efficient PLC equipment can ensure the stability and coordination of each unit on the production line. Therefore, to improve the efficiency and quality of industrial production, it is necessary to scientifically and rationally optimize each unit PLC equipment to improve the control efficiency of electrical equipment , To ensure the smooth development of industrial production activities. Combining many years of experience in electrical equipment maintenance and design, starting from the actual production situation, the author first analyzes the role and characteristics of PLC in the automatic control system, and then proposes some practical measures to optimize the design of PLC equipment to improve the efficiency of electrical equipment. Provide relevant researchers with some useful suggestions in actual design.

\section{The role and characteristics of PLC in the automation control system}

From the perspective of the overall architecture of the automation control system, PLC equipment occupies a pivotal position in the automation control system. It can be said that it is the "soul" part of the entire automation control system. Without the PLC automation control, it will be difficult to achieve orderly. "Automation" has become a misnomer. PLC can monitor the operation of electrical equipment in real time to ensure that the equipment works according to the set running track and action. In the process of PLC logic optimization, technicians should analyze various data in actual work to find the best The control node makes the entire control logic simpler and smoother. In the process of improving equipment operation efficiency and ensuring equipment operation stability, in addition to optimizing the internal program of the PLC, the wiring method of the PLC system should also be optimized at the early stage of the design, inspection work should be done, and debugging should be carried out continuously. During the rectification process, ensure that the entire control system can meet the needs of automatic control of electrical equipment, and seek the best balance between efficiency and stability.

\section{Research on the Design Practice of PLC in Automatic Control System in}

Copyright (C) 2020 Zhaosen Zhang et al.

doi: 10.18282/le.v9i5.1211

This is an open-access article distributed under the terms of the Creative Commons Attribution Non-Commercial License

(http://creativecommons.org/licenses/by-nc/4.0/), which permits unrestricted non-commercial use, distribution, and reproduction in any medium, provided the original work is properly cited. 


\section{Electrical Equipment}

\subsection{Optimize the port information transmission of the PLC system and do a good job in the equipment early warning system}

When optimizing the function of PLC equipment, we should first start with the initialization port of the system, continuously improve the construction of port data transmission, and increase the speed of data transmission. Technical staff should conduct a comprehensive monitoring of the implementation screen of the entire equipment operation to ensure electrical equipment The real-time management of the operation process can detect and deal with equipment operation problems in time, so as to avoid affecting the whole body and causing greater losses. The prerequisite for comprehensive monitoring of equipment is to make remote monitoring settings. In order to ensure the effectiveness of remote monitoring, technicians should improve the data collection channels in the equipment, collect as many data as possible on key parts of the equipment, and monitor the data. The processing efficiency is optimized and upgraded to ensure that the operation of electrical equipment can be tracked in real time and that the staff can control the overall operation of the equipment.

During the operation of electrical equipment, there may be abnormalities in any part. Some problems occur on the surface of the equipment, and some occur inside the equipment. These problems can be hardware problems or software operation problems. Therefore, some equipment failures have a certain degree of concealment. Therefore, if the equipment manager wants to understand and grasp the point of equipment failure in the first time, they should set up the failure alarm device in advance to ensure that the electrical equipment can be cut off in time after the equipment fails. When analyzing equipment failures, you should first analyze the PLC data acquisition logic analysis module, and reproduce the entire equipment operation through the displayed data, so as to find out the cause of the equipment abnormality as soon as possible. Therefore, technicians should ensure the accuracy of the PLC data, regularly upgrade the information management system, register and record various data monitoring equipment in real time, eliminate equipment failures in time, ensure the good operation of electrical equipment, and improve equipment production efficiency. Stability of equipment operation.

\subsection{The specific application of PLC technology in the automatic control system of electrical equipment}

First of all, when optimizing electrical equipment, technicians need to combine the environment of the equipment itself and the equipment's requirements for working accuracy, select the PLC model and function in a targeted manner, and evaluate the stability and reliability of the PLC in the middle of the electrical equipment. Analyze and take some effective measures to ensure the reliability of the PLC control system. For example, during the operation of the equipment, set a certain margin in advance for the relevant operating parameters to prevent excessive data errors or data overflow from causing abnormalities in the equipment; another example, in the automatic control system of PLC electrical equipment, it needs to be set in key parts Some system testing equipment performs different levels of monitoring according to the division of labor in different regions, and detects some dangerous behaviors and abnormal equipment operation to ensure the safety of the equipment.

Second, the selection and determination of the I/O address. The determination of the I/O address is the basic step of the PLC design in the electrical beaker. The technician should consider whether the number of I/O points can meet the requirements of the equipment operation, and fully consider the spare, upgrade and subsequent expansion needs of the electrical equipment before setting it. . PLC equipment actually sorts out the collected information and operates in accordance with the requirements of the program. It is the "transfer station" for data and the "receiving room" for commands. Only by doing a series of basic tasks such as data collection, storage, and output can it be done. Make the operation of electrical equipment more stable and more efficient.

Finally, the control unit design of the electrical system. The control unit in the PLC electrical control system is very important. The technicians need to set and allocate according to the actual situation, optimize the program setting, and continuously check and improve in the actual operation process to meet the actual requirements of the equipment application. Improve the stability and accuracy of electrical equipment operation. 


\section{Concluding remarks}

In summary, the development of automatic control systems for electrical equipment is inseparable from PLC technology. In specific design and application, technicians should pay attention to the collection and management of various information, and summarize and accumulate more in practice. Experience, we must be brave to innovate and dare to work hard, improve the reliability of PLC technology, make our country's electrical equipment automation system more and more perfect, and work for the welfare of the people.

\section{References}

1. Mao Bodong. Talking about the design and application of PLC in the automatic control system of electrical equipment [J]. Guangdong Science and Technology, 2019(14):154-155.

2. Zhang Chuyun. Practical analysis of the application of PLC technology in electrical equipment automation control[J]. Electronics World, 2018. (09): 54-53.

3. Zhang Na. The design and application analysis of PLC in the automatic control system of electrical equipment [J]. Electronic Technology and Software Engineering, 2015, 000(006): 180-180. 